EESTI NSV TEADUSTE AKADEEMIA TOIMETISED. 31. KOIDE FOOSIKA * MATEMAATIKA. 1982, NR. 4

ИЗВЕСТИЯ АКАДЕМИИ НАУК ЭСТОНСКОП ССР. ТОМ 31 ФИЗИКА * МАТЕМАТИКА. 1982, № 4

\title{
СЛАБО КОМПАКТНО ПОРОЖДЕННЫЕ ТЕНЗОРНЫЕ ПРОИЗВЕДЕНИЯ И ПРОСТРАНСТВА ОПЕРАТОРОВ
}

\author{
(Представил А. Хумал)
}

Исследуется, когда тензорные пронзведения $X \otimes_{\alpha} Y$ WCG-пространств $X$ и $Y$ являются WCG-пространствами. В частности, доказывается, что $X \otimes_{\alpha} Y$ является $W C G$-пространством при всех кросс-нормах $\alpha$, если одно из пространств $X$ или $Y$ является сепарабельным или обладает свойством Данфорда-Петтиса. Полученные результаты применяются для изучения слабо компактной порожденности пространств $X$-значных функций, непрерывных на компакте, интегрируемых по Бохнеру или по Петтису, а также пространств p-ядерных, p-ннтегральных, квази-р-ядерных, абсолютно p-суммирующих и компактных операторов.

1. Пусть $X$ и $Y$ - банаховы пространства (или оба реальные, или оба комплексные). Норма $\alpha$ в тензорном произведении $X \otimes Y$ называется кросс-нормой или скрещенной нормой, если $\alpha(x \otimes y)=\|x\|\|y\|$ при всех $x \in X, y \in Y$. Обозначим пополнение тензорного произведения $X \otimes Y$, наделенного кросс-нормой $\alpha$, через $X \otimes_{\alpha} Y$.

В последнее десятилетие многими авторами изучался вопрос, когда какое-либо определенное линейно-топологическое свойство (напр., рефлексивность, слабая секвенциальная полнота и пр.) пространств $X$ (или его сопряженного $X^{\prime}$ ) и $Y$ наследуется тензорными произведениями $X \otimes_{\alpha} Y$ или пространствами операторов из $X$ в $Y$ (см., напр., $\left[{ }^{1-3}\right]$ и содержащиеся там библиографии). Объектом исследования настоящей работы является свойство слабо компактной порожденности.

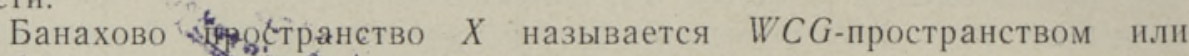
слабо компактно fрожденным пространством, если оно содержит слабо компактное"множество (согласно теореме Эберлейна-Шмульяна, слабая компактность равносильна слабой секвенциальной компактности), линейная оболочка которого плотна в $X$. Примерами $W C G$-пространств являются сепарабельные пространства, рефлексивные пространства и пространства $L_{1}=L_{1}(S, \Sigma, \mu)$ (классов эквивалентности) $\mu$-суммируемых функций на $S$, если $(S, \Sigma, \mu)$ - пространство с $\sigma$-конечной мерой.

Вопрос о слабо компактной порожденности тензорного произведения изучался Дж. Дистелем (см. $\left[{ }^{4}\right]$. или $\left.\left[{ }^{2}\right]\right)$, который показал, что $L_{1} \otimes{ }_{\gamma} X$ является $W C G$-пространством, если $(S, \Sigma, \mu)$ - пространство с конечной мерой, $X-W C G-$-пространство, а $\gamma$ - проективная кросснорма. Мы покажем, что для многих кросс-норм $\alpha$ слабо компактная порожденность пространств $X$ II $Y$ гарантирует слабо компактную порожденность $X \otimes_{\alpha} Y$, а для остальных кросс-норм укажем налагаемые на одно из пространств $X$ или $Y$ условия, при которых верна та же импликация. Из наших результатов вытекает, в частности, что в теореме Дистеля $L_{1}$ можно заменить произвольным $W C G$-пространством, обладающим свойством Данфорда-Петтиса. Результаты о тензорных произведениях применим для изучения слабо компактной порожденности операторных пространств. 
2. Введем необходимые определения и обозначения. Через $L(X, Y)$ обозначается пространство непрерывных линейных операторов из $X$ в $Y$, через $K(X, Y)$ - его подпространство компактных операторов, наделенные обычной операторной нормой. Говорят, что $X$ обладает аппроксимационным свойством, если для любого компактного подмножества $K \subset X$ и для любого числа $\varepsilon>0$ существует оператор конечного ранга $T \in L(X, X)$ такой, что $\|T x-x\|<\varepsilon$ при всех $x \in K$. Говорят, что $X$ обладает свойством Данфорда-Петтиса, если $\lim _{n} x_{n}^{\prime}\left(x_{n}\right)=0$ при всех слабо сходящихся к нулю последовательностях $\left(x_{n}\right)$ в $X$ и $\left(x_{n}^{\prime}\right)$ в $X^{\prime}$.

Пусть $\left(x_{i}\right)=\left(x_{i}\right)_{1 \leqslant i \leqslant n}-$ конечная система элементов пространства $X$. Введем следующие обозначения:

$$
\begin{gathered}
\gamma_{p}\left(x_{i}\right)=\left(\sum_{i=1}^{n}\left\|x_{i}\right\|^{p}\right)^{1 / p}, \quad 1 \leqslant p<\infty, \\
\gamma_{\infty}\left(x_{i}\right)=\sup \left\{\left\|x_{i}\right\|: 1 \leqslant i \leqslant n\right\}, \\
\beta_{p}\left(x_{i}\right)=\sup \left\{\gamma_{p}\left(x^{\prime}\left(x_{i}\right)\right): x^{\prime} \in X^{\prime},\left\|x^{\prime}\right\| \leqslant 1\right\}, \quad 1 \leqslant p \leqslant \infty .
\end{gathered}
$$

Говорят, что оператор $T \in L(X, Y)$ является абсолютно $p$-суммирующим $(1 \leqslant p \leqslant \infty)$, если существует число $\rho$ такое, что для всех конечных систем $\left(x_{i}\right)$. из $X$ выполняется неравенство $\gamma_{p}\left(T x_{i}\right) \leqslant \rho \beta_{p}\left(x_{i}\right)$. Наименьшее из а в этом неравенстве обозначается $\pi_{p}(T)$. Известно, что $\pi p$ является нормой в пространстве всех абсолютно $p$-суммирующих операторов $\Pi_{p}(X, Y)$, а $\Pi_{p}(X, Y)$, наделенное $\pi_{p}$, является банаховым. Ясно, что $\Pi_{\infty}(X, Y)=L(X, Y)$ и $\pi_{\infty}(T)=\|T\|$.

Теперь введем в $X \otimes Y$ кросс-нормы $\varepsilon_{p}$ и $g_{p}, 1 \leqslant p \leqslant \infty$. Рассматривая $X \otimes Y$ как линейное множество в $\Pi_{p}\left(X^{\prime}, Y\right)$, обозначим через $\varepsilon p$ норму в $X \otimes Y$, которую индуцирует $\pi_{p}$. Норма $\varepsilon_{\infty}$ совпадает с индуктивной кросс-нормой $\lambda$, определяемой равенством $\lambda(u)=\sup \left\{\mid\left(x^{\prime} \otimes\right.\right.$ $\left.\left.\otimes y^{\prime}\right)(u) \mid: x^{\prime} \in X^{\prime},\left\|x^{\prime}\right\| \leqslant 1, y^{\prime} \in Y^{\prime},\left\|y^{\prime}\right\| \leqslant 1\right\}$. Нормы $g_{p}$ определяются по формуле $g_{p}(u)=\inf \gamma_{p}\left(x_{i}\right) \beta_{q}\left(y_{i}\right)$, где $1 / p+1 / q=1 \quad$ (с $\left.1 / \infty=0\right)$ и inf берется по всевозможным представлениям $u=\sum_{i=1}^{n} x_{i} \otimes y_{i}$. Отметим, что $g_{1}$ совпадает с проективной кросс-нормой $\gamma$, определяемой равенством $\gamma(u)=\inf \left\{\sum_{i=1}^{n}\left\|x_{i}\right\|\left\|y_{i}\right\|: u=\sum_{i=1}^{n} x_{i} \otimes y_{i}\right\}$.

Будем говорить, что кросс-норма $\alpha$ больше, чем кросс-норма $\beta$ (или $\beta$ меньше, чем $\alpha$ ), если существует число $c>0$ такое, что $\alpha(u) \geqslant c \beta(u)$, $u \in X \otimes Y$. Известно, что $\gamma$ является наибольшей из всех кросс-норм в $X \otimes Y$, а $\lambda$ меньше, чем любая из кросс-норм $\varepsilon_{p}$ или $g_{p}$ (здесь, в частности, $c=1$ ).

Замкнутая линейная оболочка подмножества $K \subset X$ обозначается [K]. Пусть $K \subset X, L \subset Y$, а $M=\{x \otimes y: x \in K, y \in L\}$. Прибегнув к кросс-норме $\gamma$, легко проверить, что из условий $[K]=X$ и $[L]=Y$ вытекает $[M]=X \otimes_{\alpha} Y$, где $\alpha-$ произвольная кросс-норма. Пусть $X_{i}$, $Y_{i}(i=1,2)$ - банаховы пространства, $T_{i} \in L\left(X_{i}, Y_{i}\right)$ и $\alpha=\varepsilon_{p}$ или $\alpha=g_{p}(1 \leqslant p \leqslant \infty)$. Известно, что $\quad T_{1} \otimes T_{2} \in L\left(X_{1} \otimes X_{2}, \quad Y_{1} \otimes{ }_{\alpha} Y_{2}\right)$, где $T_{1} \otimes T_{2}$ определяется линейно формулой $\left(T_{1} \otimes T_{2}\right)\left(x_{1} \otimes x_{2}\right)=$ $=T_{1} x_{1} \otimes T_{2} x_{2}, \quad x_{i} \in X_{i}$, а $X_{1} \otimes X_{2}$ наделено $\alpha$. Продолжение по непрерывности $T_{1} \otimes T_{2}$ на $X_{1} \otimes{ }_{\alpha} X_{2}$ обозначим также через $T_{1} \otimes T_{2}$. Заметим, что если $T_{i}\left(X_{i}\right)$ плотно в $Y_{i}(i=1,2)$, то $\left(T_{1} \otimes T_{2}\right)\left(X_{1} \otimes \alpha X_{2}\right)$ плотно в $Y_{1} \otimes{ }_{\alpha} Y_{2}$.

Оператор $T \in L(X, Y)$ называется $p$-интегральным $(1 \leqslant p<\infty)$, если существует положительная конечная мера $\mu$ на слабо* компактном единичном шаре $U^{0} \subset X^{\prime}$ такая, что $Q T$ факторизуется следующим образом:

$$
X \stackrel{i}{\rightarrow} C\left(U^{0}\right) \stackrel{j}{\rightarrow} L_{p}\left(U^{0}, \mu\right) \stackrel{\stackrel{s}{\rightarrow}}{\rightarrow} Y^{\prime \prime}
$$


где $i, j$ и $Q: Y \rightarrow Y^{\prime \prime}$ - соответствующие канонические вложения и $S-$ линейный оператор с $\|S\| \leqslant 1$. Пространство р-интегральных операторов $I_{p}(X, Y)$ является банаховым с нормой $i_{p}(T)=\inf \mu\left(U^{0}\right)^{1 / p}$, где inf берется по всевозможным факторизациям QT. Банаховы пространства $p$-ядерных и квази-р-ядерных операторов из $X$ в $Y$ определяются так же, как и в $\left[{ }^{5}\right]$, и обозначаются соответственно $N_{p}(X, Y)$ и $Q N_{p}(X, Y)$.

Bсе необходимые факты о $W C G$-пространствах можно найти в $\left[{ }^{6}\right]$, об аппроксимационном свойстве и о свойстве Данфорда-Петтиса, например, в $\left[{ }^{2}\right]$, о тензорных произведениях - в $\left[{ }^{7-9}\right]$, об операторных пространствах - в $\left[{ }^{5,10,11}\right]$.

3. Перейдем к вопросу о слабо компактной порожденности тензорных произведений. Начнем с двух простых наблюдений:

1) если $X \otimes_{\alpha} Y$ является WCG-пространством, то $X \otimes_{\beta} Y-W C G$-пространство для всех кросс-норм $\beta$, меньших, чем $\alpha$;

2) если $X \otimes{ }_{\alpha} Y-W C G$-пространство при кросс-норме а, больией, чем

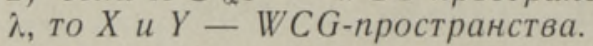

Наблюдение 1) вытекает из следующего очевидного факта: если $X-W C G$-пространство и область значений $T(X)$ оператора $T \in L(X, Y)$ плотна в $Y$, то и $Y$ является $W C G$-пространством. Что же касается 2), то, согласно 1), его достаточно доказать при $\alpha=\lambda$. Так как $X \otimes_{\lambda} Y$ канонически изометрически изоморфно подпространству $L\left(X^{\prime}, Y\right)$ (см. $\left.\left[{ }^{8}\right]\right)$, то $T: A \rightarrow A x^{\prime}$ будет при произвольном фиксированном $x^{\prime} \neq 0$ линейной непрерывной сюръекцией из $X \otimes_{\lambda} Y$ на $Y$ и, следовательно, $Y$ - $W C G$-пространство. А слабо компактная порожденность $X$ следует, в силу только что доказанного, из изометрической изоморфности $X \otimes_{\lambda} Y$ и $Y \otimes_{\lambda} X$.

Поскольку $\gamma=g_{1}$ - наибольшая из всех кросс-норм, то представляет интерес прежде всего слабо компактная порожденность $X \otimes{ }_{\gamma} Y$. Последнее тензорное произведение в общем не является $W C G$ пространством, даже тогда, когда $X$ и $Y$ - рефлексивные пространства с аппроксимационным свойством. Например, если $S$ - несчетное множество, то $l_{2}(S) \otimes_{\gamma} l_{2}(S)$ не является $W C G$-пространством, поскольку оно содержит топологически дополняемое подпространство, изоморфное $l_{1}(S)$. Мы имеем следующий «положительный» результат:

Те орема 1. Если X и них сепарабельно или обладает свойством Данфорда-Петтиса, то $X \otimes_{\alpha} Y$ является WCG-пространством при всех кросс-нормах $\alpha$.

Доказательство достаточно проводить для $\alpha=\gamma$. Ввиду изометрического изоморфизма между $X \otimes_{\gamma} Y$ и $Y \otimes_{\gamma} X$ можем предполагать, что ограничения налагают на $Y$.

Рассмотрим случай, когда $Y$ сепарабельно. Пусть $\left\{z_{1}, z_{2}, \ldots\right\}$, $z_{n} \neq 0,-$ плотное множество в $Y$. Пусть, далее, $K$ - слабо компактное множество, порождающее $X, L=\left\{0, y_{1}, y_{2}, \ldots\right\}$, где $y_{n}=z_{n} /\left(n\left\|z_{n}\right\|\right)$, и $M=\{x \otimes y: x \in K, y \in L\}$. Так как $[K]=X$ и $[L]=Y$, то. $[M]=$ $=X \otimes_{\gamma} Y$. Покажем, что $M$ слабо компактно в $X \otimes_{\gamma} Y$. Рассмотрим последовательность $\left(u_{n}\right)$ в $M$. Ясно, что $\left(u_{n}\right)$ содержит подпоследовательность вида $\left(x_{k} \otimes y_{n_{k}}\right)$ или $\left(x_{n} \otimes y\right)$. Ввиду ограниченности $\left(x_{k}\right)$ ясно, что $\left(x_{k} \otimes y_{n_{k}}\right)$ сходится к нулю (заметим, что $0 \in M$ ) по норме $\gamma$. Рассмотрим $\left(x_{n} \otimes y\right)$. В силу слабой компактности $K \subset X$ существует подпоследовательность $\left(x_{n_{k}}\right)$, сходящаяся слабо к некоторому элементу $x \in K$. Следовательно, при произвольном операторе $A \in L\left(Y, X^{\prime}\right)$ имеем $(A y)(x)=\lim _{k}(A y)\left(x_{n_{k}}\right)$. Так как $\left(X \otimes{ }_{\gamma} Y\right)^{\prime}$ отождествляется с $L\left(Y, X^{\prime}\right)$ (см. $\left.\left[{ }^{8}\right]\right)$, то $\left(x_{n_{k}} \otimes y\right)$ сходится слабо к $x \otimes y$ в $X \otimes{ }_{\gamma} Y$. 
Пусть $Y$ обладает свойством Данфорда-Петтиса. Обозначим через $K$ и $L$ слабо компактные множества, порождающие соответственно $X$ и $Y$. Тогда $X \otimes_{\gamma} Y=[M]$, где $M=\{x \otimes y: x \in K, y \in L\}$. Рассмотрим произвольную последовательность $\left(x_{n} \otimes y_{n}\right)$ в $M$. В силу слабой компактности множеств $K \subset X$ и $L \subset Y$ можем считать, не ограничивая общности, что $\left(x_{n}\right)$ и $\left(y_{n}\right)$ сходятся слабо соответственно к некоторым элементам $x \in K$ и $y \in L$. Тогда при любом операторе $A \in L\left(X, Y^{\prime}\right)$ последовательность $\left(A\left(x_{n}-x\right)\right)$ сходится слабо к нулю в $Y^{\prime}$ и, в силу свойства Данфорда-Петтиса пространства $Y$, получаем, что $\lim _{n}\left(A\left(x_{n}-x\right)\right)\left(y_{n}-y\right)=0$. Так как $(A x)\left(y_{n}\right) \rightarrow_{n}(A x)(y)$ и $\left(A x_{n}\right)(y)=\left(A^{\prime} Q y\right)\left(x_{n}\right) \rightarrow n\left(A^{\prime} Q y\right)(x)=(A x)(y)$ (где $Q-$ каноническое вложение $Y^{\prime}$ в $\left.Y^{\prime \prime}\right)$, то $\lim _{n}\left(A x_{n}\right)\left(y_{n}\right)=(A x)(y)$. И поскольку $\left(X \otimes{ }_{\gamma} Y\right)^{\prime}$ отождествляется с $L\left(X, Y^{\prime}\right)\left(\right.$ см. $\left.\left[{ }^{8}\right]\right)$, то $\left(x_{n} \otimes y_{n}\right)$ сходится слабо к $x \otimes y$ в $X \otimes{ }_{\gamma} Y$.

3 а мечание 1. В частном случае, когда одно из пространств - например $Y$ - не только сепарабельно, но и обладает базисом, утверждение теоремы 1 следует из результата Шадвика $\left[{ }^{12}\right]$ : банахово пространство $Z$ с шаудеровым разложением $\left(P_{n}\right)$ (т. е. с последовательностью ненулевых непрерывных проекторов $P_{n}$ в $Z$, удовлетворяющей условиям $P_{n} P_{m}=0, n \neq m$, и $z=\sum_{n=1}^{\infty} P_{n} z, \quad z \in Z$ ) является $W C G$ пространством, если каждое $P_{n}(Z)-W C G$-пространство. Действительно, легко проверить, что $P_{n}=I \otimes p_{n}$ (где $I$ - тождественный оператор в $X$ и $p_{n}$ - одномерные проекторы в $Y$, связанные естественным образом с базисом $\left(e_{n}\right)$ пространства $\left.Y\right)$ образуют шаудерово разложение для $Z=X \otimes_{\gamma} Y$, причем $P_{n} Z$ изоморфно $X$.

Используя наблюдение 2) и тот факт, что $L_{1}$ и $C(\Omega)$ обладают свойством Данфорда-Петтиса, получаем следующие три следствия (в них $X$ - банахово пространство).

Следств и е 1 . Пусть $(S, \Sigma, \mu)$ - пространство с б-конечной мерой. Пространство $X$-значных $\mu$-интегрируемых по Бохнеру функций $L_{1}(X)$ является слабо компактно порожденным тогда $и$ только тогда, когда $X-W C G$-пространство.

Для доказательства заметим, что $L_{1}=L_{1}(S, \Sigma, \mu)$ есть $W C G$-пространство и $L_{1}(X)$ изометрически изоморфно $L_{1} \otimes_{\gamma} X$ (см. $\left.\left[{ }^{8}\right]\right)$.

3 а мечание 2. Следствие 1 в случае конечной меры было получено Дж. Дистелем (см. $\left[{ }^{4}\right]$ или $\left.\left[{ }^{2}\right]\right)$; он доказал его посредством теории мер, опираясь на теорему факторизации Дейвиса-Фигеля-Джонсона-Пелчинского $\left[{ }^{13}\right]$.

Следствне 2. Пусть $(S, \Sigma, \mu)$ - пространство с конечной мерой и $P_{1}(\mu, X)$ - пространство $\mu$-измеримых интегрируемых по Петтису $X$-значных функций. Пополнение $P_{1}(\mu, X)$ является WCG-пространством тогда и только тогда, когда $X-W C G$-пространство.

Для доказательства достаточно заметить, что пополнение $P_{1}(\mu, X)$ изометрически изоморфно $L_{1} \otimes{ }_{\lambda} X$ (см., напр., $\left.\left[{ }^{2}\right]\right)$.

Напомним $\left[{ }^{14,6}\right]$, что отделимый компакт $\Omega$ называется компактом Эберлейна, если $\Omega$ гомеоморфен слабо компактному подмножеству некоторого банахова пространства.

Следстви е 3 . Пусть $\Omega$ - отделлимый компакт. Пространство непрерывных $X$-значных функций $C_{X}(\Omega)$ является слабо компактно порожденным тогда и только тогда, когда $X-$ WCG-пространство $и \Omega-$ компакт Эберлейна.

Доказ ательст в о использует изометрический изоморфизм между $C_{X}(\Omega)$ и $C(\Omega) \otimes_{\lambda} X \quad\left(\right.$ см. $\left.\left[{ }^{8}\right]\right)$, а также результат Амира и Линденштраусса $\left[{ }^{14}\right]$, который обобщается следствием 3 , что $C(\Omega)$ является 
$W C G$-пространством тогда и только тогда, когда $\Omega-$ компакт Эберлейна.

Как видно из следующей теоремы, для «достаточно маленьких» кросс-норм тензорное произведение будет всегда $W C G$-пространством. Те орема 2. Если $X$ и $Y-W C G$-пространства, то $X \otimes_{\alpha} Y$ является WCG-пространством при всех кросс-нормах $\alpha$, меньших, чем $g_{2}$.

Доказ ательство достаточно проводить для $\alpha=g_{2}$. Так как $X$ и $Y$ - WCG-пространства, то вследствие вышеназванной теоремы факторизации (см. $\left[{ }^{13}\right]$ или $\left.\left[{ }^{6}\right]\right)$ существуют рефлексивные пространства $Z, W$ и операторы $T \in L(Z, X), S \in L(W, Y)$ такие, что $T(Z)$ и $S(W)$ плотны соответственно в $X$ и $Y$. Поскольку $T \otimes S \in L\left(Z \otimes_{g_{2}} W, X \otimes_{g_{2}} Y\right)$ и $(T \otimes S)\left(Z \otimes g_{2} W\right)$. плотно в $X \otimes_{g_{2}} Y$, то достаточно установить слабо компактную порожденность $Z \otimes_{g_{2}} W$. Но последнее пространство, по результатам П. Сафара $\left[{ }^{15}\right]$, даже рефлексивно.

Т ео рем а 3. Пусть Х и

а) $p=1$ и одно из пространств $X$ или $Y$ сепарабельно или обладает свойством Данфорда-Петтиса,

б) $1<p<2$ и одно из пространств $X$ или $Y$ сепарабельно или обладает свойством Данфорда-Петтиса или же сопряженное $X^{\prime} \kappa X$ обладает аппроксимационным свойством,

в) $2 \leqslant p \leqslant \infty$,

то $X \otimes g_{p} Y$ является WCG-пространством тогда и только тогда, когда Х и - WCG-пространства.

Доказ ател̈ьство. Необходимость ясна из наблюдения 2).

Достаточность. Поскольку $g_{p}, \quad 1 \leqslant p \leqslant \infty$, образуют убывающее семейство $\left[{ }^{9}\right]$, то в силу теорем 1 и 2 остается доказать слабо компактную порожденность $X \otimes_{g_{p}} Y$ лишь в случае, когда $1<p<2$ и $X^{\prime}$ обладает аппроксимационным свойством. Так как для $Y$ найдется рефлексивное пространство, которое отображается линейно и непрерывно на плотное в $Y$ множество, то без ограничения общности можем считать $Y$ рефлексивным. Пусть $K, L$ и $M$ - те же, что и в доказательстве второй части теоремы 1 , и пусть из произвольной последовательности множества $M$ выделена подпоследовательность $\left(u_{n}\right)=\left(x_{n} \otimes y_{n}\right)$ такая, что $\left(x_{n}\right)$ и $\left(y_{n}\right)$ сходятся слабо соответственно к $x$ в $X$ и к $y$ в $Y$. Чтобы показать слабую сходимость $\left(u_{n}\right)$ к $u=x \otimes y$, напомним [ $\left.{ }^{9}\right]$, что $\left(X \otimes_{g_{p}} Y\right)^{\prime}$ и $\Pi_{q}\left(Y, X^{\prime}\right)(1 / p+1 / q=1)$ канонически изометрически изоморфны. Значит, рассматривая $u_{n}$ и $u$ как элементы $\left(\Pi_{q}\left(Y, X^{\prime}\right)\right)^{\prime}$, нужно доказать, что $\left(u_{n}\right)$ сходится к $u$ всюду на $\Pi_{q}\left(Y, X^{\prime}\right)$. Для этого воспользуемся теоремой Банаха-Штейнгауза. Заметим, во-первых, что нормы функционалов $u_{n}$ ограничены, так как $\left\|u_{n}\right\|=g_{p}\left(u_{n}\right)=\left\|x_{n}\right\|\left\|y_{n}\right\|$. Во-вторых, $\left(u_{n}\right)$ сходится к $u$ на $Y^{\prime} \otimes X^{\prime} \subset \Pi_{q}\left(Y, X^{\prime}\right)$, так как $u_{n}\left(y^{\prime} \otimes x^{\prime}\right)=y^{\prime}\left(y_{n}\right) x^{\prime}\left(x_{n}\right) \rightarrow n y^{\prime}(y) x^{\prime}(x)=u\left(y^{\prime} \otimes x^{\prime}\right)$ при всех $y^{\prime} \in Y^{\prime}$ и $x^{\prime} \in X^{\prime}$. Остается показать, что $Y^{\prime} \otimes X^{\prime}$ плотно в $\Pi_{q}\left(Y, X^{\prime}\right)$. Но в силу рефлексивности $Y$ имеем $\Pi_{q}\left(Y, X^{\prime}\right)=Q N_{q}\left(Y, X^{\prime}\right)$ (см. $\left.\left[{ }^{10}\right]\right)$, причем их нормы равны. И так как $Y^{\prime} \otimes X^{\prime}$ плотно в $Q N_{q}\left(Y, X^{\prime}\right)$, поскольку $X^{\prime}$ обладает аппроксимацнонным свойством $\left[{ }^{5}\right]$, то $Y^{\prime} \otimes X^{\prime}$ плотно в $\Pi_{q}\left(Y, X^{\prime}\right)$.

Т еор ем а 4. B предположениях теоремы 3, где в а) ещце допускается, что X - рефлексивное пространство, обладающее аппроксимационным свойством, имеет место утверждение: $X \otimes_{\varepsilon_{p}} Y$ - WCG-пространство тогда и только тогда, когда $X$ и $Y$ - WCG-пространства.

Доказательство необходимости то же, что и в теореме 3 .

Достаточность. Пусть сначала выполнены предположения теоре- 
мы 3. Так как $\varepsilon_{p}$ меньше, чем $g_{p}$ (см. $\left.\left[{ }^{9}\right]\right)$, то в силу наблюдения 1) результат вытекает из теоремы 3. Докажем слабо компактную порожденность $X \otimes_{\varepsilon_{1}} Y$ в предположениях рефлексивности и аппроксимационного свойства $X$. Как и в доказательстве теоремы 3 , достаточно рассматривать случай, когда $Y$ рефлексивно. Поскольку $X^{\prime}$ рефлексивно и обладает аппроксимационным свойством (см. $\left[{ }^{8}\right]$ или $\left[{ }^{2}\right]$ ), то, по результату Гордона-Льюнса-Резерфорда $\left[{ }^{16}\right]$, пространство $\Pi_{1}\left(X^{\prime}, Y\right)$ рефлексивно. И так как $X \otimes_{\varepsilon_{1}} Y$ канонически изометрически изоморфно подпространству $\Pi_{1}\left(X^{\prime}, Y\right)$, то и оно рефлексивно.

4. Следующие результаты о слабо компактной порожденности операторных пространств вытекают из теорем 3 и 4, а также из того факта, что $X^{\prime}$ и $Y$ являются $W C G$-пространствами, если одно из пространств $N_{p}(X, Y), I_{p}(X, Y), Q N_{p}(X, Y), \Pi_{p}(X, Y)$ нли $K(X, Y)$ слабо компактно порождено. Последнее утверждение доказывается аналогично наблюдению 2) в разделе $3:$ при фиксированных $x \neq 0$ и $y^{\prime} \neq 0$ рассматриваются операторы $T$ на $Y$ и $S$ на $X^{\prime}$, определяемые равенствами $T A=A x$, $S A=A^{\prime} y^{\prime}$, где $A-$ произвольный элемент из данного операторного пространства и $A^{\prime} \in L\left(Y^{\prime}, X^{\prime}\right)-$ сопряженный к $A$ оператор.

След ств и е 4 . Пусть $X и Y-$ банаховы пространства $и 1 \leqslant p \leqslant \infty$. Если сопряженное пространство $X^{\prime}$ и $Y$ соответственно удовлетворяют тем же условиям, что и $Х$ и $Y$ в теореме 3 , то $N_{p}(X, Y)$ является WCGпространством тогда и только тогда, когда $X^{\prime}$ и $Y$ - WCG-пространства.

Для доказательства отметим, что если $X^{\prime}$ и $Y-W C G$-пространства, то, по теореме $3, X^{\prime} \otimes{ }_{g_{p}} Y-W C G$-пространство. Но так как существует сюръекция $T \in L\left(X^{\prime} \otimes_{g_{p}} Y, N_{p}(X, Y)\right) \quad\left[{ }^{9}\right], \quad$ то и $N_{p}(X, Y)$ является $W C G$-пространством.

Следствие 5. Пусть выполнены предположения следствия 4 u $1 \leqslant p<\infty$. Если $X^{\prime}$ обладает аппроксимационным свойством или же $Y$ топологически дополняемо в $Y^{\prime \prime}$, то $I_{p}(X, Y)$ является WCG-пространством тогда и только тогда, когда $X^{\prime}$ и $Y$ - WCG-пространства.

3 а меч ан и е 3 . Если $X^{\prime \prime}$ обладает аппроксимацнонным свойством, то и $X^{\prime}$ обладает этим свойством (см. $\left[{ }^{8}\right]$ или $\left.\left[{ }^{2}\right]\right)$.

Доказ ательство. Заметим, что если $X^{\prime}-W C G$-пространство, то оно обладает свойством Радона-Никодима (см. $\left[{ }^{2}\right]$ или $\left.\left[{ }^{6}\right]\right)$. Но если $X^{\prime}$ обладает и аппроксимационным свойством, то, как отмечает С. Хейнрих (см. $\left[{ }^{3}\right]$, с. 172 , или $\left.\left[{ }^{1}\right]\right), I_{p}(X, Y)=N_{p}(X, Y), 1 \leqslant p<\infty$, причем эти пространства изоморфны, поскольку их нормы сравнимы. То же равенство имеет место и в случае, когда $Y$ топологически дополняемо в $Y^{\prime \prime}$ (см. $\left[{ }^{1}\right]$ или $\left.\left[{ }^{3}\right]\right)$. Остается применить следствие 4.

Следств и е 6. Пусть Х и или $Y$ обладает аппроксимационным свойством, $и$ пусть $1 \leqslant p<\infty$. Если $X^{\prime}$ и $Y$ соответственно удовлетворяют тем же условиям, что и $X$ и $Y$ в теореме 4, то следующие утверждения равносильны:

1) $Q N_{p}(X, Y)-W C G$-пространство,

2) $\Pi_{p}(X, Y)-W C G$-пространство,

3) $X^{\prime}$ и $Y-W C G$-пространства.

Д оказ ательство. Если $X^{\prime}$ и $Y-W C G$-пространства, то, по теореме $4, X^{\prime} \otimes_{\varepsilon_{p}} Y-W C G$-пространство. Как мы отмечали в предыдущем доказательстве, $X^{\prime}$ обладает свойством Радона-Никодима, поэтому $Q N_{p}(X, Y)=\Pi_{p}(X, Y), 1 \leqslant p<\infty$ (см. [ $\left.{ }^{1}\right]$ или $\left.\left[{ }^{3}\right]\right)$, причем их нормы равны. Известно $\left[{ }^{5}\right]$, что аппроксимационное свойство $X^{\prime}$ или $Y$ гарантирует плотность $X^{\prime} \otimes Y$ в $Q N_{p}(X, Y)$. Значит, $X^{\prime} \otimes_{\varepsilon_{p}} Y=\Pi_{p}(X, Y)$ и, следовательно, $\Pi_{p}(X, Y)$ и $Q N_{p}(X, Y)-W C G$-пространства. 
Следствие 7. Если $X, и Y$ - банаховы пространства такие, что $X^{\prime}$ или $Y$ обладает аппроксимационным свойством, то $K(X, Y)-W C G$ пространство тогда и только тогда, когда $X^{\prime}$ и $Y$ - WCG-пространства.

Для доказательства воспользуемся теоремой 4 , учитывая, что $\varepsilon_{\infty}=\lambda$ и, в наших предположениях, пространства $X^{\prime} \otimes_{\lambda} Y$ и $K(X, Y)$ канонически изометрически изоморфны $\left[{ }^{8}\right]$.

3 а м еч ани е 4. По результату Н. Дж. Калтона $\left[{ }^{17}\right]$ о нерефлексивности $L(X, X)$ ясно, что $K(X, X)$ не является рефлексивным, если $X-$ рефлексивное несепарабельное пространство, обладающее аппроксимационным свойством, поскольку в этом случае $(K(X, X))^{\prime \prime}=\left(X^{\prime} \otimes_{2} X\right)^{\prime \prime}=$ $=\left(X \otimes{ }_{\gamma} X^{\prime}\right)^{\prime}=L(X, X)$ '(см. $\left.\left[{ }^{8}\right]\right)$. Однако, по следствию 7, пространство $K(X, X)$ слабо компактно порождено.

\section{ЛИТЕРАТУ РА}

1. Макаров Б. М., Сам а рский В. Г., Функц. анализ и его прнлож., 15 , № 3 , 89-90 (1981).

2. Dieste l, J., Uh l, J. J., Jr., Vector measures. Math. Surveys, 15, Amer. Math. Soc., Providence-Rhode Island, 1977.

3. He in rich, S., Serdica Bulg. Math. Publ., 3, 168-175 (1977).

4. D i e ste l, J., Proc. Amer. Math. Soc., 48, № 2, 508-510 (1975).

5. Persson, A., P i e t s c h, A., Stud. Math., 33, 19-62 (1969).

6. Д и стель Дж., Геометрия банаховых пространств. Избранные главы, Киев, «Вища школа», 1980.

7. Д э й М. М., Нормированные линейные пространства, М., ИЛ, 1961.

8. Grothendieck, A., Mem. Amer. Math. Soc., 16, 1-191 (1955).

9. S a p h a r, P., Stud. Math., $38,71-100$ (1970).

10. Pers s o n, A., Stud. Math., 33, 213-222 (1969).

11. P i e t s c h, A., Stud. Math., 28, 333-353 (1967).

12. Ch a d w ic k, J. J. M., Bull. Austral. Math. Soc., 6, 133-144 (1972).

13. Davis, W. J., Fig ie 1, T., Johnson, W. B., Pelczynski, A., J. Funct. Anal., $17,311-327$ (1974).

14. A m i r, D., Lindenstrauss, J., Ann. Math., 88, 35-46 (1968).

15. S a p h a r, P., Israel J. Math., 13, 379-399 (1972).

16. Gordon, Y., Lew is, D. R., Retherford, J. R., J. Funct. Anal., 14, 85-129 (1973)

17. Kalto n, N. J., Math. Ann., 208, 267-278 (1974).

Тартуский государственный университет

\section{Поступила в редакцию} 18/II 1982

Eve OJA

\section{NORGALT KOMPAKTSE OSAHULGA GENEREERITUD TENSORKORRUTISED JA OPERAATORITE RUUMID}

On uuritud, millal WCG-ruumide $X$ ja $Y$ tensorkorrutised $X \otimes_{\alpha} Y$ on WCG-ruumid. Saadud tulemusi on rakendatud Bochneri mōttes integreeruvate, kompaktil pidevate või Pettise mōttes integreeruvate $X$-väärtustega funktsioonide ruumidele, samuti $p$-tuuma-, $p$-integraal-, kvaasi- $p$-tuuma-, absoluutselt $p$-summeerivate ja kompạktsete operaatorite ruumidele. 


\section{WCG-PRODUITS TENSORIELS ET ESPACES D'OPÉRATEURS}

Soient $X$ et $Y$ deux espaces de Banach. Une norme $\alpha$ sur $X \otimes Y$ est dite tensorielle si $a(x \otimes y)=\|x\|\|y\|, x \in X, y \in Y$. Le complété de $X \otimes Y$ muni de $\alpha$ est noté $X \otimes_{\alpha} Y$.

Soient $X$ et $Y$ deux $W C G$-espaces de Banach. Nous démontrons (cf. théorème 2), que $X \otimes_{\alpha} Y$ est $W C G$ pour les normes tensorielles $\alpha$ plus petites que $g_{2}$ (cf. $\left[{ }^{9}\right]$ ), et (cf. théorème 1) si l'un des espaces $X$ ou $Y$ est séparable ou possède la propriété de Dunford-Pettis alors $X \otimes_{\alpha} Y$ est $W C G$ pour toutes les normes tensorielles $\alpha$. Nous caractérisons la $W C G$-propriété de $X \otimes_{\alpha} Y$ pour les normes tensorielles $\alpha=g_{p}$ et $\alpha=g_{p} \backslash=\varepsilon_{p}, 1 \leqslant p \leqslant \infty$, étudiées dans $\left[{ }^{9,15}\right]$. Par exemple, dans le cas où le dual $X^{\prime}$ a la propriété d'approximation et $1<p<2$, nous obtenons que $X \otimes_{g_{p}} Y$ est $W C G$ si, et seulement si $X$ et $Y$ sont $W C G$ (cf. théorème 3).

En application de ces, résultats nous étudions la $W C G$-propriété des espaces de fonctions à valeurs dans $X$ Bochner intégrables, continues sur un compact ou Pettis intégrables, et des espaces d'opérateurs $p$-nucléaires, $p$-intégraux, quasi-p-nucléaireș, absolument $p$-sommants et compacts. 